\title{
Research on Self-adaptive TFRC Congestion Control of Streaming Media in Wireless Network
}

\author{
Xing $\mathrm{Li}^{1}$, Pin Wang ${ }^{1,} *$ and Limin Shao ${ }^{2}$ \\ ${ }^{1}$ School of Mathematics and Information Science, Guangxi College of Education, \\ Nanning 530023, China \\ ${ }^{2}$ College of Mechanical and Electrical Engineering, Agricultural University of \\ Hebei, Baoding, 071001, China \\ Corresponding Author: Wang Pin, School of Mathematics and Information \\ Science, Guangxi College of Education, Nanning, 530023, China
}

\begin{abstract}
Transport layer protocol, for lack of effective congestion control, made an irrational bandwidth utilization ratio between UDP and TCP. Especially for streaming media transport which is easily to cause the network congestion. TFRC (TCP-friendly rate control protocol) congestion control mechanism had solved the problem effectively. However, in the high bit error rate environment of wireless network, TFPC would mistake the bit error for congestion lost packet that degrades throughput excessively. Concerning this issue, this paper put forward a self-adaptive TFRC mechanism which distinguishes congest and bit error effectively by distinguishing parameters, and adjusts the transmission rate dynamically. Simulation result reveals that self-adaptive TFRC mechanism can use link efficiently, reduce delay variation and meet the live transmission requirement of streaming media in wireless network.
\end{abstract}

Keywords: Streaming media, wireless network, TFRC, congestion control, selfadaptive

\section{Introduction}

With the growth of network and multimedia technology, streaming media application has become a significant online video play mode. However, for its high smoothness demand of message transmission and delay variation sensitivity, streaming media business brings huge challenge to network bandwidth. It is simple and unpractical to increase the bandwidth unlimitedly, especially under the wireless network environment. How to use the existing bandwidth effectively and reasonably, reduce the bandwidth waste caused by congest has become one of the current network research hotspots.

In TCP/IP protocol system, there are two kinds of end to end transport layer control protocols: TCP and UDP. TCP's congestion avoidance and retransmission mechanism ensure the fairness of bandwidth allocation and the accuracy of data stream. Meanwhile, it produces a large network delay, so TCP is not suitable for real-time service transmission of streaming media. UDP as a connectionless, best effort unreliable transport protocol can use network bandwidth efficiently under the preferable network transmission environment, reduce the transmission delay of streaming media, so it is more suitable than TCP as a transport protocol for streaming media data. However, in case of network bandwidth shortage or multiple data streams competition, UDP exposed its shortcoming. First, its connectionless mode results in unawareness of network congestion. Even in a congested network environment, it sent data on and on which accelerates the congestion. Second, UDP 
is unfair to TCP. Slow start machine makes UDP to occupy more bandwidth in a congested situation unfairly because of the concession of TCP. Throughput rate is reduced, and congestion is worsened. Therefore, a new streaming media transmission control protocol which can use network bandwidth efficiently and solve congestion control is needed

\section{Improvement of TFRC Congestion Control Mechanism}

\subsection{TFRC Protocol Basic Working Principle}

The TFRC protocol is proposed by S. Floyd, which is designed primarily for streaming media transmission characteristics based on the rate control of TCP Friendly congestion control protocol. Through the congestion control like TCP, the protocol can effectively prevent the collapse caused by network congestion, and avoid fast reduction of the transmission speed in the congestion of the environment which forms a shaking of the throughput rate, and make the effectively usage of the link bandwidth. TFRC is more fair of TCP flow comparing with the UDP protocol, and under the condition of a multiple data streams, it can be more smoothly.to adjust the rate and is suitable for streaming media applications.

Among the specific algorithm implementation, TFRC uses throughput model of TCP - RENO protocol to adjust the sending rate [5], as is shown in equation (1).

$$
T=\frac{S}{R \sqrt{\frac{2 b p}{3}}+\frac{1}{m} t_{-} R T O\left(3 \frac{3 b p}{8}\right) p\left(1+32 p^{2}\right)}
$$

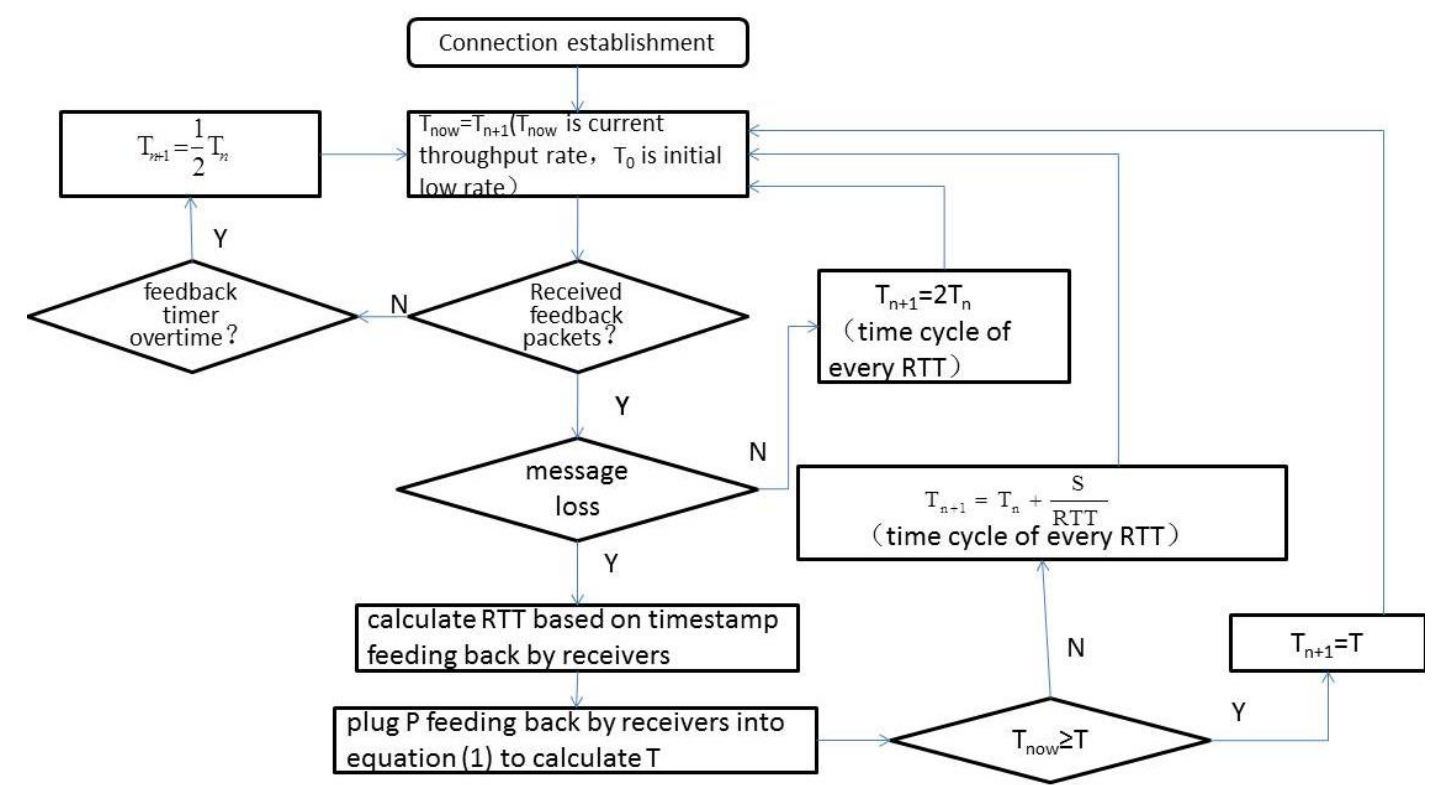

Figure 1. The Control Flow Chart of the Sender Throughput Rate of TCPTFRC Protocol

Where, $\mathrm{T}$ is the throughput rate (units: BPS), $\mathrm{S}$ is the sending packet size (unit: B), R is the loopback time RTT (unit: S), p is the packet loss event rate, and t_RTO is the focus timeout clock, which is usually set to $4 *$ RTT. B is the single TCP ACK receiving message number, if $b$ is set to 2 , it means the receiver sends an ACK response when receiving two packets. Because the majority of applications adopt 
barely delayed response, so generally the value of $\mathrm{b}$ is set to $1 . \mathrm{m}$ is the adjust parameters with desirable reference value of 1.5 . It can be seen in the equation that the sender use the packet loss probability and RTT from the receiving end to adjust the sending rate, and the TFRC protocol specific work process is shown in Figure 1:

It can be seen from the diagram that the key parameters through the throughput rate to adjust the sending rate are the packet loss rate and the loopback time, both of which are closely related to network congestion situation. TFRC has class TCP slow start work, avoids congestion and implements the TCP friendly, but simultaneously won't quickly enter a state of sending rate in half. Through the statistics of packet loss event, it is concluded that the corresponding rate is reduced with more efficient usage of bandwidth.

\subsection{TFRC Problems in Wireless Environment}

The primary design of TFRC is aimed at the cable network environment.In the cable network environment, the link layer has high stability, so the main reason the packet drop is congestion. With the popularity of wireless network, the TFRC needs to run in the wireless environment, and due to the characteristic of the wireless, the main reasons of the network packet loss is not only from congestion, but also the high bit error rate of wireless transmission [7].

In the above TFRC model, the average packet loss event $\mathrm{p}$ is defined as shown in equation (2).

$$
\begin{aligned}
& p=\frac{\sum_{i=O}^{n} w_{i}}{\sum_{i=O}^{n} A_{i} w_{i}} \\
& w_{i}=e^{-\frac{i}{c}}
\end{aligned}
$$

Where, the $\mathrm{Ai}$ is the recently interval of a packet loss event as the difference between the two groups in the serial number of the two consecutive packet packet loss events, wi is the loss event interval weight of the i packet event, take which as a one dimensional gaussian kernel function as shown in equation (2). It shows that the newer packet loss events, the more affect the value increases of $p$. The option value of $\mathrm{n}$ is 8 according to a recommended TFRC protocol. If the value of $\mathrm{n}$ is too small, there will be poor TCP friendliness, otherwise, if the value of $n$ is too large, there will be lack usage of bandwidth efficient .From the equation (2), if there is error of packet loss in the packet loss event as inserting in error packet loss in the congestion packet, the loss event error packet will be mistaken for congestion, packet loss to improve the $\mathrm{p}$ value, the contrast of errata calculation process is shown in Figure 2.

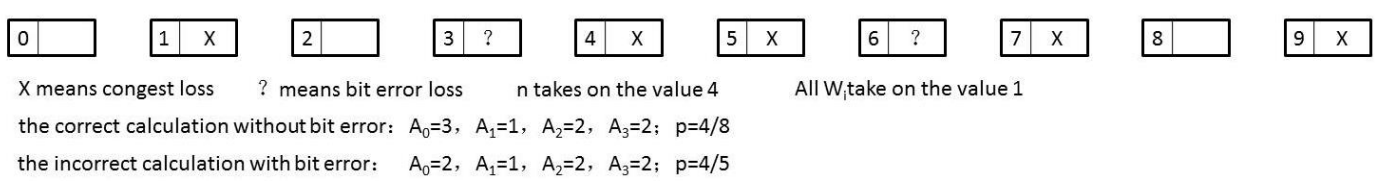

Figure 2. The Contrast of Errata Calculation when there Exists Error

As is shown in the equation (1), the increase of the $p$ value will cause the sender lower the transmission speed. The sending rate reduction is helpless in lowering bit 
error rate in the wireless network, but reduces the network bandwidth utilization, increase the time delay, and lead to a fall in the quality of streaming media.

\subsection{The Distinction between the Packet Loss Types under the Wireless Environment}

Under the wireless environment, the network is in a state of congestion or in a wireless error state can be discrimination by time delay and the delay jitter. Here, the standard reference document RFC3550 is referred to calculate the packet delay and packet delay jitter[8], for the case of a group i, the S (I)is defined as the packet sent time, $\mathrm{R}$ (i) is the group receiving time, the packet end-to-end delay t_delay (I)is shown in the equation (3), and the group delay jitter between I - 1 D (I, I - 1)) in group $\mathrm{i}$ and group $\mathrm{i}-1$ is shown in equation (4).

$$
\begin{gathered}
t_{-} \operatorname{delay}(i)=R(i)-S(i) \\
D(i, i-1)=t_{-} \operatorname{delay}(i)-t_{-} \operatorname{delay}(i-1)
\end{gathered}
$$

When $\mathrm{D}(\mathrm{I}, \mathrm{I}-1)=0$, it indicates the group delay between the $\mathrm{i}-1$ group and the i group is equal, and the network has good stable condition. When the D (I, I - 1) > 0 , it indicates there are great delay in transit in the group $i$, and the reason may come from the link layer retransmission error or the transport layer of congestion in line. Since the delay caused by the error of the link is smaller than the cause by the congestion of relatively transport layer [9], the direct and effective way to distinct these two kinds of reason for the delay is determined through a threshold, it is judged as error state when the delay is less than the threshold, and it is judged as the network

\subsection{The Congestion Control Mechanism of an Improved Adaptive TFRC}

As is shown through the above analysis that, the rational TFRC congestion control in wireless network environment improvement needs to be able to identify the causes of congestion, and adaptively take corresponding velocity control strategy to solve the problem according to the reasons.

First, it is needed to find a reasonable threshold for the judgment of the congestion reasons. At the receiving end, a packet of one-way delay is represented as ROTT, and a long-term sample ROTT value weighted average calculation can get a weighted average ROTTMEAN, the value of which can better reflect the current network status, so as a threshold. But in the process of sample adoption, it is needed to pay attention that out-of-order packets can be created in retransmission to eliminate the error produced by the link layer errors, and then ROTT values will produce larger dithering. As the wobble is not caused by congestion of transport layer or direct wireless error, and have great impact on the ROTTMEAN value calculation, so the mechanism of variance is adopted to eliminate this kind of outof-order error packet jitter in the improvement scheme. In the scheme, variance of each packet's ROTT value calculation are done, only the variance ROTT value which is eligible can be adopted as the basis to determine the state of the network, and to be allowed as the average calculation sample, thereby to eliminate random error. The improved ROTTMEAN calculation method as is shown in equation (5).

$$
\begin{aligned}
& \text { ROTTMEAN }_{n}=\mu \times \text { ROTTMEAN }_{n-1}+(1-\mu) \text { ROTT }_{n} \\
& \mu=\left\{\begin{array}{l}
1,\left(\left(\text { ROTT }_{n}-\text { ROTTMEAN }_{n-1}\right)^{2} \geq \eta \sigma_{n-1}^{2}\right) \\
0.95,\left(\left(\operatorname{ROTT}_{n}-\text { ROTTMEAN }_{n}\right)^{2}<\eta \sigma_{n-1}^{2}\right.
\end{array}\right.
\end{aligned}
$$


Where, ROTTn represents the actual one-way delay value of the $\mathrm{n}$ packet, ROTTMEANn represents weighted average of the first n package. $\sigma_{n-1}^{2}$ Represents the weighted variance of the recent n-1 packet of ROTT value, in which the determination coefficient $\eta$ plays a role in regulating. If the value of $\eta$ is set too small, there will increase the possibility of packet loss as out-of-order packets. If the value of $\eta$ is set too large, there will increase the packet loss as the possibility of congestion packet loss or error packet loss. The values of $\eta$ is between 1.55 and 1.75, experience value of 1.65 from the experimental results is adopted in this scheme. Take the value of ROTTMEAN as the threshold to distinguish the congestion packet loss and wireless packet loss. According to the TCP Veno equation, the value of adjustment factor should take 3 [6] from the Ai standard in the equation (2), therefore the introduced adjusting factor function will be the section function considering 3 as the segment point. When the ROTTn is greater than ROTTMEANn-1, the network state is determined as the congestion state, and the value of adjustment factor should be taken within the range of [1], so as to reduce the packet loss interval to increase the packet loss event rate, and to lower transmission speed. According to the ideas of making full use of bandwidth based on TFRC, the lowered speed should be more moderate, so in this condition the piecewise function should be defined as exponential function. When ROTTn is less than ROTTMEANn-1, the network state is determined as the non-congestion state, or the error packet loss, at this time the value of adjustment factor should take within the range of [3], thereby to increase the packet loss interval to reduce the packet loss event rate, and to improve the transmission speed, Since bandwidth space is available at this time, the transmission speed can be improved rapidly, so the condition of piecewise function is defined as a linear function. The improved adjustment factor function of $\mathrm{A}(\mathrm{i})$ is definitions as shown in equation (6).

$$
A(i)=\left\{\begin{array}{l}
e^{-\left(\text {ROTT }_{i}-\text { ROTTMEAN }_{i-1}\right)^{2} /\left(2 \sigma^{2}\right)}, \text { ROTT }_{i}>\text { ROTTMEAN }_{i-1} \\
-2 \frac{\text { ROTT }_{i}}{\text { ROTTMEAN }_{i-1}}+3, \text { ROTT }_{i} \leq \text { ROTTMEAN }_{i-1}
\end{array}\right.
$$

Where, $O$ is a minimum, when the delay is greater than the threshold as $A(i) \in$ [1], the packet loss interval reduces. When the delay is greater than the threshold as A (I) A(i) $\in$ [3], the packet loss interval increases. The improved packet loss event probability $\mathrm{p}^{*}$ is as shown in equation (7).

$$
p^{*}=\frac{\sum_{i=O}^{n} w_{i}}{\sum_{i=O}^{n} A(i) A_{i} w_{i}}
$$

Put the value of $\mathrm{p}^{*}$ into the equation (1), the improved adaptive TFRC throughput model is obtained is as shown in formula (8).

$$
T=\frac{S}{R \sqrt{\frac{2 b p^{*}}{3}}+\frac{1}{m} t_{-} R T O\left(3 \frac{3 b p^{*}}{8}\right) p^{*}\left(1+32 p^{2}\right)}
$$

The improved throughput rate adjustment process is: 1, By collecting sample data at the receiving end, the threshold is obtained according to the formula (5).2, Comparing the packet delay time with the threshold to get the packet loss causes and the level of congestion.3, According to the formula (6) and formula (7) packet 
loss event rate are obtained.4,According to the equation (8), the new throughput is get using the method of flow chart of congestion control according to the throughput in Figure 1. Finally the adaptive TFRC congestion control in wireless network is realized of the streaming media transmission. For convenience of expression, Adaptive - TFRC is called the in the experiment.

\section{The Simulation Results And Analysis}

\subsection{Setting of Simulation Experiment}

The validation, comparison and analysis of network performance of TFRC and improved adaptive TFRC under the environment of wireless are done based on the NS2 network simulation tools. The set topology structure of Simulation experiment is as shown in Figure 3.

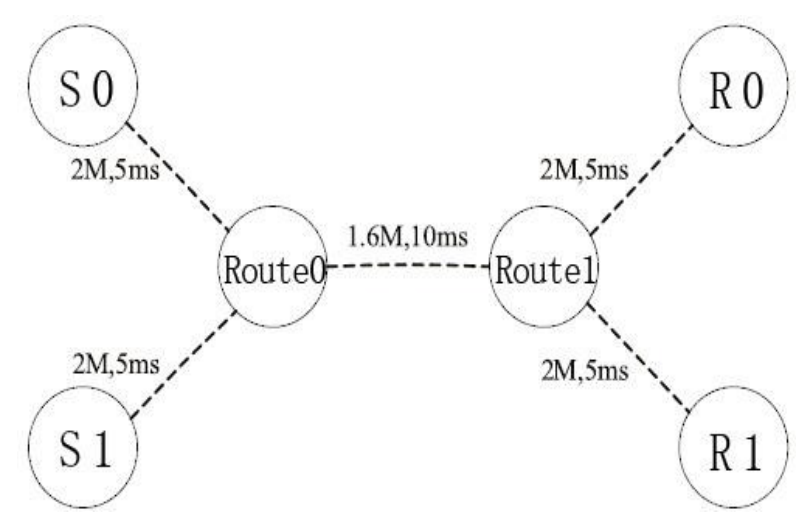

Figure 3. The Topology Structure of Simulation Experiment

In the Figure 3, the dumbbell topology is adopted, S0 and S1are chosen for sending node, R0, R1 are the receiving node, Route 0 , Route 1 are routing nodes, S0 and R0 binds with TCP protocol as background, S1 and R1 binds with TFRC protocol agent and the Adaptive-TFRC agent. The bandwidth between Route0 and Route 1 is $1.6 \mathrm{M}$, with delay for $10 \mathrm{~ms}$, which comparing with $2 \mathrm{M}$ bandwidth and $10 \mathrm{~ms}$ delay of other link forms the bottleneck link and congestion. The queuing strategy of R0 and R1 is set for DropTail queue management. The wireless error packet loss rate is set for $1 \%$.

To compare two groups of experiments, the TCP flow is operated in both groups between S0 and R0, the experiments start from 0 seconds, and TFRC flow between $\mathrm{S} 1$ and R1 will start in 5 seconds in order to compare TFRC friendliness, and experimental time is $100 \mathrm{~s}$. The first group is testing with standard TFRC protocol, the second group is testing with the Adaptive-TFRC. The throughput and delay jitter are mainly analyze in two groups of simulation tests.

\subsection{The Analysis and Comparison of Throughput}

The comparisons of the throughput performance of two experiments are as shown in Figure 4. 

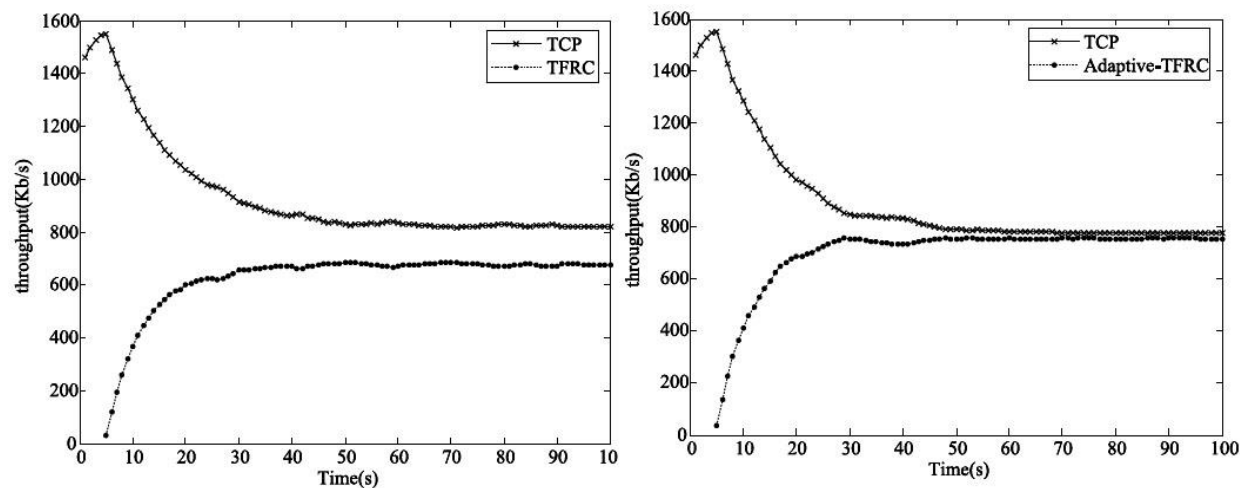

\section{Figure 4. The Comparisons of the Throughput Performance of TFRC and Adaptive-TFRC}

It can be seen from the diagram that both agreements have good friendly and form the both enjoy of bandwidth comparing with the TCP. But the Adaptive TFRC is more fully on the efficiency of bandwidth. From the data view, the average throughput of TCP and TFRC are $615 \mathrm{~KB} / \mathrm{S}$ and $930 \mathrm{~KB} / \mathrm{S}$ with ratio of 0.661 and remaining bandwidth of $55 \mathrm{~KB} / \mathrm{S}$ in the left figure. The average throughput of TCP and TFRC are $690 \mathrm{~KB} / \mathrm{S}$ and $900 \mathrm{~KB} / \mathrm{S}$ with ratio of 0.767 and remaining bandwidth of $10 \mathrm{~KB} / \mathrm{S}$ in the right figure. The Adaptive - TFRC in the presence of wireless packet loss can better share bandwidth with TCP, and reduced the residual bandwidth of $45 \mathrm{~KB} / \mathrm{S}$, which suggests that it makes the use of the bandwidth more fully. This is because the Adaptive - TFRC can distinguish the type of packet loss, avoid the interference of the wireless error packet loss to adaptively regulate the throughput rate with full use of bandwidth.

\subsection{Analysis and Comparison of Delay Jitter}

The analysis and comparison of two experiments of delay jitter are shown in Figure 5
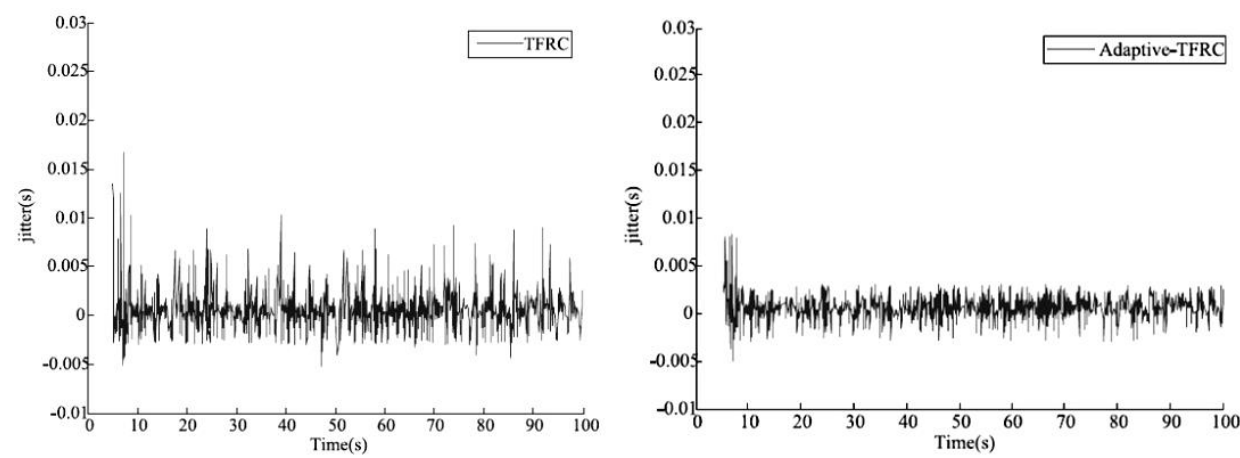

Figure 5. The Comparison of Delay Jitters with TFRC and Adaptive-TFRC

It can be seen from the diagram, the delay jitter of Adaptive - TFRC is smaller and more concentrate. From the data view, the variance of throughput rate is $0.004 \mathrm{~S}$ in the left figure, and variance of throughput rate is $0.003 \mathrm{~S}$ in the right figure, so jitter value of the Adaptive-TFRC reduces about 33\%. That is because the Adaptive TFRC can be more accurate and timely adjustment to the throughput rate, make transport more smooth and more conducive to streaming media. 


\section{Conclusion}

The research aiming at the problems of running streaming media in the wireless environment is studied. The working principle of TFRC and the problem of the exist of wireless error are analyzed, and an improved adaptive TFRC congestion control mechanism are proposed. Experimental results show that the Adaptive-TFRC can ensure the basis of friendliness of TCP to adaptively distinct packet loss type, make full use of bandwidth to decrease the delay jitter, and is in favor of the operation of the media in wireless environment.

\section{Acknowledgements}

This work is supported by the Guangxi Provincial Natural Science Research Project for Universities (No.: KY2015YB338), and Characteristic Professional Project fund of the Education Department of Guangxi, China (No.: GXTSZY277), and Foundation of Guangxi Educational Committee, China (No.: 2014JGA268), and the Guangxi Department of Education 2015 Reform Project of Higher Education of Guangxi (No. 2015JGZ172).

\section{References}

[1] S.X. Liu, "The Internet Congestions Control Algorithms Research and Simulation Analysis", Chengdu: Southwest Jiaotong University, (2005).

[2] X.W. Yao, W.L. Wang and S.H. Yang, "PABM-EDCF: Parameter Adaptive Bi-directional Mapping Mechanism for Video Transmission over WSNs", Multimedia Tools and Applications, doi: 10.1007/s11042-011-0934-7, (2011).

[3] S. Floyd, M. Handley, J. Padhye and J. Widmer, "RFC 5348: TCP Friendly Rate Control (TFRC): Protocol Specification, RFC5348", (2008).

[4] Z.G. Chen, X.H. Deng and L.M. Zhang, "TCP Friendly Streaming Media Transmission Improvement System in Wireless Network", Journal of communications, vol. 28, no. 5, (2007), pp. 22-28.

[5] P. Wen, J. Cao and Y. Li, "Design of High- performance Networked Real time Control Systems", Control Theory \& Applications, vol. 1, no. 5, (2007), pp. 1329-1335.

[6] B. Zhou, “An Enhancement of TFRC over Wireless Networks", IEEE Trans. on Multimedia, vol. 8, no. 5, (2007), p. 1045.

[7] W.T. Sun and Q. Guo, "A Kind of Streaming Media Transmission Mechanism Suitable for Wireless Network", Journal of computer applications, vol. 29, no. 1, (2009), pp.12-15.

[8] H. Schulzrinne and S. Casnerl, "RFC 3550, RTP: A Transport Protocol for Real-time Applications", (2003).

[9] C. Song, P.C. Cosman and G.M. Voelker, "End-to-end Differentiation of Congestion and Wireless".

[10] B. Zhou, C.P. Fu and D.M. Chiu, "A Simple Throughput Model for TCP Veno // IEEE I CC 06. June.

[11] P. Xiao, R.C. Wang and L.J. Sun, "Wireless Network Congestion Control Mechanism Research Based on the TCP Friendly", Computer science, no. 7, (2010), pp. 51-53.

[12] H. Yuan, M.K. Du and C.B. Li, "A Suitable TFRC Protocol for Wireless Network Streaming Media Transmission Improvement", Journal of Harbin University of Technology, no.4, (2011), pp. 40-43.

[13] W.L. Wang, X.W. Zhang and X.W. Yao, "2013 Adaptive TFRC Mechanism Based on Streaming Media Transmission in Wireless Network", Application computer systems, no. 7, (2013), pp.161-167. 